\title{
Path Integral Solution of the Kramers Problem
}

\author{
A. N. Drozdov* and M. Morillo \\ Física Teórica, Universidad de Sevilla, Apartado de Correos 1065, Sevilla 41080, Spain
}

(Received 30 September 1996)

\begin{abstract}
An iterative method to generate a discrete path integral solution of the Kramers problem is presented. It is based on a straightforward derivation of the functional formalism from the underlying Langevin equations. The method is rather simple and systematic and allows us to analytically evaluate the short time propagator up to and including terms of fourth order in a time increment $\tau$. This means a significant reduction of the number of time steps $N$ that are necessary to obtain convergent results for a given net increment $t=N \tau$. [S0031-9007(96)02050-9]
\end{abstract}

PACS numbers: 05.40.+j, 02.50.Ey

Path integral representations are commonly used in studies of physical problems whose dynamics are described in terms of Schrödinger, Fokker-Planck, or Langevin equations [1]. The starting point for their derivation is the fact that the propagator for a finite time $t$ can be factored into a product of $N$ propagators, each one propagating the system for a shorter time interval $\tau=t / N$,

$$
\begin{aligned}
P\left(\mathbf{q}, t \mid \mathbf{q}^{0}\right)= & \int d \mathbf{q}^{N-1} \cdots \int d \mathbf{q}^{1} P\left(\mathbf{q}, \tau \mid \mathbf{q}^{N-1}\right) \\
& \cdots P\left(\mathbf{q}^{1}, \tau \mid \mathbf{q}^{0}\right)
\end{aligned}
$$

where $\mathbf{q}^{T}=\left\{q_{1}, \ldots, q_{n}\right\}$. The above equation is actually exact for any number of time slices $N$. The only advantage of breaking up the propagator according to Eq. (1) is that we can use on the right-hand side of this equation, instead of the exact propagator $P\left(\mathbf{q}^{i+1}, \tau \mid \mathbf{q}^{i}\right)$, its approximation $P_{(k)}\left(\mathbf{q}^{i+1}, \tau \mid \mathbf{q}^{i}\right)$,

$$
P\left(\mathbf{q}^{i+1}, \tau \mid \mathbf{q}^{i}\right)=P_{(k)}\left(\mathbf{q}^{i+1}, \tau \mid \mathbf{q}^{i}\right)+O\left(\tau^{k+1}\right),
$$

which needs to be valid at least to first order in $\tau,(k=1)$. The discrete path integral thus obtained,

$$
\begin{aligned}
P\left(\mathbf{q}, t \mid \mathbf{q}^{0}\right)= & \int d \mathbf{q}^{N-1} \cdots \int d \mathbf{q}^{1} P_{(k)}\left(\mathbf{q}, \tau \mid \mathbf{q}^{N-1}\right) \\
& \cdots P_{(k)}\left(\mathbf{q}^{1}, \tau \mid \mathbf{q}^{0}\right)+O\left(t^{k+1} / N^{k}\right),
\end{aligned}
$$

expresses the distribution function for any (arbitrary) time $t$ in terms of the known short time propagator.

For many years the path integral has provided a powerful formal tool for deriving both perturbative and nonperturbative systematic approximations in quantum and statistical mechanics. Numerical applications have also become increasingly important during the last decade and have often led to new physical results not obtainable by other means [2]. Until recently, however, this approach failed to treat stochastic processes with noninvertible diffusion matrices, as there were no discrete path integral representations for these processes, mainly due to the "singularity" problem associated with the integration measure.

Here we will deal with one of the most extensively studied models of such a type, the so-called Kramers model. The dynamics described by the model is that of a Brownian particle moving in a potential $\mathcal{U}(x)$. It is governed by the Fokker-Planck equation (also called the Klein-Kramers equation) for the probability density of finding the particle at time $t$ at position $x$ with velocity $v$. In dimensionless variables it reads

$$
\begin{aligned}
\partial_{t} P(x, v, t)= & {\left[-v \partial_{x}-G(x) \partial_{v}+\gamma \partial_{v}\left(v+\varepsilon \partial_{v}\right)\right] } \\
& \times P(x, v, t),
\end{aligned}
$$

where $\gamma$ is the friction coefficient, $\varepsilon$ is a measure of the noise intensity, $G(x)=-\mathcal{U}^{\prime}(x)$, and the prime denotes differentiation with respect to $x$. It may be noted that this equation, first applied to investigate the Brownian motion [3] and the rates of chemical reactions [4], is now largely employed, in various generalized forms, in physics, chemistry, and biology to study systems driven by noise and friction [5]. We also note that the diffusion matrix associated with Eq. (4) does not possess an inverse. The latter property implies that the integration measure of the standard path integral representation available in the literature for the multidimensional Fokker-Planck equation becomes a singular ( $\delta$-function-like) quantity. This makes impossible the numerical evaluation of the path integral and hinders considerably obtaining explicit solutions or approximations of the Wenzel-Kramers-Brillouin type.

In the present Letter we outline a method for generating precise discrete path integral representations of the propagator, which is applicable regardless of whether the diffusion matrix is singular or not. One might, at first, believe that this issue should have been settled long ago, mainly because of its continuous usefulness in many problems ranging from chemical physics to communication theory. To the best of our knowledge, however, there are no general path integral solutions of such a type, other than those derived by splitting the original operator $L$ into a linear contribution $L_{0}$ and an anharmonic correction $L_{1}$, and approximating each short time evolution operator by a product of exponentials [6]. The disadvantage of this approach is that its utility is generally restricted to the case $k \leq 2$. Although formally possible to construct, any higher order approximants involve either polynomials of order $k$ of the operators $L_{0}$ and $L_{1}$ [7] or negative coefficients [8], making them rather impractical for stochastic 
processes. In practice, though, one would like for the short time propagators to be accurate for an order in $\tau$ as high as possible, in order to keep the number of integration variables in Eq. (3) as small as possible (for a given net time increment $t$ ). We also note that it is the singularity of the diffusion matrix which prevents us from making use of an efficient power series expansion formalism developed in a recent series of papers by Drozdov [9].

Our aim is to lift this restriction and to provide a systematic strategy that permits the analytic evaluation of the short time propagator at least to fourth order in $\tau$. The key idea is the same as in the formal (continuous time) path integral method which is based on a straightforward derivation of the functional formalism from Langevin equations without the necessity of introducing noncommuting operators into the discussion [10]. To keep the presentation simple we restrict ourselves to Eq. (4). At the end we shall indicate the steps needed to adapt the method to an arbitrary (either additive or multiplicative) multidimensional stochastic process. The Langevin equations associated with Eq. (4) read

$$
\dot{X}=V, \quad \dot{V}+\gamma V-G(X)=f(t),
$$

where $f(t)$ is a Gaussian white noise normalized to

$$
\langle f(t)\rangle=0, \quad\langle f(t) f(s)\rangle=2 \gamma \varepsilon \delta(t-s),
$$

and where capitals are used in order to distinguish the stochastic variables $(X, V)$ from those in Eq. (4). The continuous time path integral solution for the above process is easily obtained by using Eq. (5) to transform the probability density functional for the noise, which is given by

$$
P[f(t)]=C \exp \left[-\frac{1}{4 \gamma \varepsilon} \int_{0}^{t} d s f^{2}(s)\right],
$$

with $C$ being a normalization constant, to the probability density functional for the coordinate $x$ :

$$
\begin{aligned}
P[x(t)]=C J_{f}[x(t)] \exp \{ & -\frac{1}{4 \gamma \varepsilon} \int_{0}^{t} d s \\
& \left.\times[\ddot{x}+\gamma \dot{x}-G(x)]^{2}\right\} .
\end{aligned}
$$

The integrand in Eq. (8) is understood to be evaluated at the time $s$, while $J_{f}[x(t)]$ denotes the Jacobian of the transformation over the same time interval $(0, t)$ from the $f(t)$ realizations to the $x(t)$ realizations. In the limit of vanishingly small noise, $\gamma \varepsilon \rightarrow 0$, Eq. (8) can be evaluated by the method of steepest descents. The paths which domi- nate the integrals correspond to those trajectories which minimize the exponent in Eq. (8) under the conditions $x(t)=x, v(t)=v, x(0)=x_{0}, v(0)=v_{0}[10]$. For finite $\gamma \varepsilon$, a numerical evaluation of the path integral is generally required. Numerical implementation of Eq. (8) is a far from simple task.

Here, we suggest an alternative procedure which consists of two steps. The first step is to obtain an approximate solution, for a short time $\tau$, of the stochastic process $Q(t)=(X(t), V(t))$. The most common method of doing so relies on the integration of the Langevin equations from $t_{i}=i \tau$ to $t_{i}+\tau$ and the expansion of the drift coefficients in a Taylor series about the prepoint $Q\left(t_{i}\right)=$ $\left(X\left(t_{i}\right), V\left(t_{i}\right)\right)$ [11]. When applied to Eq. (5) this yields

$$
\begin{aligned}
X\left(t_{i}+\tau\right)= & X_{i}+\int_{t_{i}}^{t_{i}+\tau} d s V(s), \\
V\left(t_{i}+\tau\right)= & V_{i}-\gamma \int_{t_{i}}^{t_{i}+\tau} d s V(s) \\
& +\sum_{m=0} \frac{G_{i}^{(m)}}{m !} \int_{t_{i}}^{t_{i}+\tau} d s\left[X(s)-X_{i}\right]^{m} \\
& +\int_{t_{i}}^{t_{i}+\tau} d s f(s),
\end{aligned}
$$

where we set $Q_{i}=Q\left(t_{i}\right)$ and $G_{i}^{(m)}=G^{(m)}\left(X_{i}\right)$. The above equations are then solved iteratively by using the following recurrence relations:

$$
\begin{aligned}
X_{(k)}\left(t_{i}+\tau\right)= & X_{i}+\int_{t_{i}}^{t_{i}+\tau} d s V_{(k-1)}(s) \\
V_{(k)}\left(t_{i}+\tau\right)= & V_{i}-\gamma \int_{t_{i}}^{t_{i}+\tau} d s V_{(k-1)}(s) \\
& +\sum_{m=0}^{k-1} \frac{G_{i}^{(m)}}{m !} \int_{t_{i}}^{{ }^{t_{i}+\tau}} d s\left[X_{(k-1)}(s)-X_{i}\right]^{m} \\
& +\int_{t_{i}}^{t_{i}+\tau} d s f(s) .
\end{aligned}
$$

In this Letter we will not go beyond fourth order in $\tau$. Neglecting terms of order higher than $\tau^{4}$, one obtains

$$
\begin{aligned}
& X_{i+1}-x_{d}\left(X_{i}, V_{i}, \tau\right)=Y_{i+1}, \\
& V_{i+1}-v_{d}\left(X_{i}, V_{i}, \tau\right)=U_{i+1},
\end{aligned}
$$

where the quantities $x_{d}\left(X_{i}, V_{i}, \tau\right)$ and $v_{d}\left(X_{i}, V_{i}, \tau\right)$ are given by the expressions

$$
\begin{aligned}
x_{d}\left(X_{i}, V_{i}, \tau\right)= & X_{i}+\tau V_{i}+\frac{\tau^{2}}{2}\left(G_{i}-\gamma V_{i}\right)+\frac{\tau^{3}}{6}\left[\gamma^{2} V_{i}-\gamma G_{i}+V_{i} G_{i}^{\prime}\right] \\
& +\frac{\tau^{4}}{24}\left[\gamma^{2} G_{i}-\gamma^{3} V_{i}+G_{i}^{\prime}\left(G_{i}-2 \gamma V_{i}\right)+V_{i}^{2} G_{i}^{\prime \prime}\right], \\
v_{d}\left(X_{i}, V_{i}, \tau\right)= & V_{i}+\tau\left(G_{i}-\gamma V_{i}\right)+\frac{\tau^{2}}{2}\left[\gamma^{2} V_{i}-\gamma G_{i}+V_{i} G_{i}^{\prime}\right]+\frac{\tau^{3}}{6}\left[\gamma^{2} G_{i}-\gamma^{3} V_{i}+G_{i}^{\prime}\left(G_{i}-2 \gamma V_{i}\right)+V_{i}^{2} G_{i}^{\prime \prime}\right] \\
& +\frac{\tau^{4}}{24}\left[\gamma^{4} V_{i}-\gamma^{3} G_{i}+G_{i}^{\prime}\left(3 \gamma^{2} V_{i}-2 \gamma G_{i}+V_{i} G_{i}^{\prime}\right)+V_{i} G_{i}^{\prime \prime}\left(3 G_{i}-4 \gamma V_{i}\right)+V_{i}^{3} G_{i}^{\prime \prime \prime}\right],
\end{aligned}
$$


and $\left(Y_{i+1}, U_{i+1}\right)$ stand for

$$
\begin{aligned}
Y_{i+1}= & F_{1}(\tau)-\gamma F_{2}(\tau)+\left(\gamma^{2}+G_{i}^{\prime}\right) F_{3}(\tau), \\
U_{i+1}= & F_{0}(\tau)-\gamma F_{1}(\tau)+\left(\gamma^{2}+G_{i}^{\prime}\right) F_{2}(\tau) \\
& -\left(\gamma^{3}+2 \gamma G_{i}^{\prime}\right) F_{3}(\tau) \\
& +\frac{G^{\prime \prime}}{2} \int_{t_{i}}^{t_{i}+\tau} d s F_{1}(s)\left[F_{1}(s)+2 s V_{i}\right]
\end{aligned}
$$

with

$$
F_{m}(\tau)=\int_{0}^{\tau} d t_{m} \int_{0}^{t_{m}} d t_{m-1} \cdots \int_{0}^{t_{2}} d t_{1} \int_{0}^{t_{1}} d t_{0} f\left(t_{0}\right) .
$$

Equation (11) relates the two sets of variables $\left(X_{N}, V_{N}, \ldots, X_{1}, V_{1}\right) \quad$ and $\quad\left(Y_{N}, U_{N}, \ldots, Y_{1}, U_{1}\right)$. As the functions involved in Eq. (11) are all evaluated at the prepoint $\left(X_{i}, V_{i}\right)$, the Jacobian of the transformation is equal to unity in this case. Therefore, the short time propagator of the discretized $(X, V)$ realization reads

$$
P_{(4)}\left(x_{i+1}, v_{i+1}, \tau \mid x_{i}, v_{i}\right)=R_{(4)}\left(y_{i+1}, u_{i+1}, \tau\right),
$$

where $R$ is the probability distribution of the fluctuating terms $(Y, U)$.

Thus, the second step of the present method is the derivation of the statistical properties of these terms. Since $f(t)$ is Gaussian and has a zero mean, the $F$ 's are also Gaussian with correlation properties [11]

$$
\begin{aligned}
\left\langle F_{m}(\tau)\right\rangle & =0, \\
\left\langle F_{m}(s) F_{n}(\tau)\right\rangle & =2 \gamma \varepsilon \frac{s^{m+1}}{m !} \sum_{i=0}^{n} \frac{s^{i}(\tau-s)^{n-i}}{i !(n-i) !(m+i+1)} \\
(s<\tau) . &
\end{aligned}
$$

Using Eqs. (6) and (16) shows after lengthy but simple calculations that the first cumulants of the noise terms are given by

$$
\begin{aligned}
\left\langle Y_{i+1}\right\rangle= & 0, \quad\left\langle U_{i+1}\right\rangle=v_{s}\left(x_{i}, \tau\right)=\frac{\gamma \varepsilon}{12} \tau^{4} G_{i}^{\prime \prime}, \\
\left\langle Y_{i+1}^{2}\right\rangle= & \sigma_{x x}(\tau)=\frac{2}{3} \gamma \varepsilon \tau^{3}\left(1-\frac{3}{4} \gamma \tau\right), \\
\left\langle Y_{i+1} U_{i+1}\right\rangle= & \sigma_{x v}\left(x_{i}, \tau\right) \\
= & \gamma \varepsilon \tau^{2}\left[1-\gamma \tau+\frac{1}{12} \tau^{2}\left(7 \gamma^{2}+4 G_{i}^{\prime}\right)\right], \\
\left\langle U_{i+1}^{2}\right\rangle= & \sigma_{v v}\left(x_{i}, v_{i}, \tau\right) \\
= & 2 \gamma \varepsilon \tau\left[1-\gamma \tau+\frac{1}{3} \tau^{2}\left(2 \gamma^{2}+G_{i}^{\prime}\right)\right. \\
& \left.-\frac{1}{12} \tau^{3}\left(4 \gamma^{3}+5 \gamma G_{i}^{\prime}-3 v_{i} G_{i}^{\prime \prime}\right)\right],
\end{aligned}
$$

while all cumulants of higher order than 2 vanish. The latter property implies that the pair stochastic process $(Y, U)$ remains Gaussian up to and including terms of order $\tau^{4}$. This immediately yields

$$
\begin{aligned}
P_{(4)}\left(x_{i+1}, v_{i+1}, \tau \mid x_{i}, v_{i}\right)=\left[4 \pi^{2} \sigma\left(x_{i}, v_{i}, \tau\right)\right]^{-1 / 2} \exp \{ & -\frac{\sigma_{v v}\left(x_{i}, v_{i}, \tau\right)}{2 \sigma\left(x_{i}, v_{i}, \tau\right)}\left[x_{i+1}-x_{d}\left(x_{i}, v_{i}, \tau\right)\right]^{2}+\frac{\sigma_{x v}\left(x_{i}, \tau\right)}{\sigma\left(x_{i}, v_{i}, \tau\right)} \\
& \times\left[x_{i+1}-x_{d}\left(x_{i}, v_{i}, \tau\right)\right]\left[v_{i+1}-v_{d}\left(x_{i}, v_{i}, \tau\right)-v_{s}\left(x_{i}, \tau\right)\right] \\
& \left.-\frac{\sigma_{x x}(\tau)}{2 \sigma\left(x_{i}, v_{i}, \tau\right)}\left[v_{i+1}-v_{d}\left(x_{i}, v_{i}, \tau\right)-v_{s}\left(x_{i}, \tau\right)\right]^{2}\right\}
\end{aligned}
$$

where $\sigma\left(x_{i}, v_{i}, \tau\right)=\sigma_{x x}(\tau) \sigma_{v v}\left(x_{i}, v_{i}, \tau\right)-\sigma_{x v}^{2}\left(x_{i}, \tau\right)$, and where the matrix elements $\sigma_{k j}$ and the average $v_{s}\left(x_{i}, \tau\right)$ are given by Eq. (17). It will also be recalled that the quantities $x_{d}\left(x_{i}, v_{i}, \tau\right)$ and $v_{d}\left(x_{i}, v_{i}, \tau\right)$ are given by Eq. (12).

As evidenced by Eq. (17), the range of validity of the above approximation for the propagator is rather sensitive to the friction coefficient. The time increment $\tau$ should always be taken small enough in the high friction limit $\gamma \rightarrow \infty$ so that the integration measure remains positive, and may be taken large enough otherwise. We also note that the utility of the present technique in deriving precise and easily evaluatable short time propagators for Eq. (4) is restricted to $3 \leq k \leq 4$. For lower $k$ the matrix element $\sigma_{x x}(\tau)$ becomes equal to zero and the singularity problem is met in this case. While for $k \geq 5$, higher order cumulants of the pair stochastic process $(Y, U)$ are no longer equal to zero and non-
Gaussian corrections are needed. The latter complicates considerably the analytic evaluation of the short time propagator. Beyond these limits, however, the procedure outlined above preserves its significance as a convenient starting point for constructing higher order stochastic Runge-Kutta algorithms.

Substitution of Eq. (18) into Eq. (3) yields the discrete path integral representation of the Kramers problem we are looking for. This representation is indeed rigorous and provides a powerful tool for obtaining both perturbative and nonperturbative approximations. But its strength and limitations when treating systematically nonlinear systems have yet to be explored. From a computational point of view, however, the discrete path integral already has much to offer. The advantages of the present solution become more evident if one compares it with the continuous time integral representation, Eq. (8). The latter is purely formal and, therefore, no simpler to implement 
numerically than the original Fokker-Planck equation [Eq. (4)]. In contrast, the discrete path integral permits one to devise very efficient algorithms for numerical simulations which cannot be developed in terms of partial [Eq. (4)] or stochastic [Eq. (5)] differential equations. For example, a powerful method could be based on the global (Monte Carlo sampling) techniques available for the evaluation of multidimensional integrals [12]. The most appealing feature of this approach is that it does not require explicit reference to the distribution function and thus avoids storing large matrices. As, however, storage requirements are not so dramatic for two dimensional systems, one can also evaluate the present discrete path integral iteratively by quadrature. The latter approach may be very efficient in terms of speed if one takes advantage of fast Fourier transforms [13]. We are presently working on this problem.

Finally, we outline the steps necessary to adapt the present method to an arbitrary stochastic process $\mathbf{Q}^{T}(t)=$ $\left\{Q_{1}(t), \ldots, Q_{m}(t)\right\}$ governed by the difference equation

$$
\begin{aligned}
\mathbf{Q}\left(t_{i}+\tau\right)-\mathbf{Q}\left(t_{i}\right)= & \int_{t_{i}}^{t_{i}+\tau} d s \mathbf{G}[\mathbf{Q}(s)] \\
& +\int_{t_{i}}^{t_{i}+\tau} d s \mathbf{B}[\mathbf{Q}(s)] \mathbf{f}(s),
\end{aligned}
$$

where $\mathbf{f}^{T}(t)=\left\{f_{1}(t), \ldots, f_{m}(t)\right\}$ is a Gaussian white noise with statistical properties

$$
\left\langle f_{i}(t)\right\rangle=0, \quad\left\langle f_{i}(t) f_{j}(s)\right\rangle=\delta_{i j} \delta(t-s) .
$$

Expanding the drift and diffusion coefficients about the prepoint and using the same procedure as above, one arrives at a functional representation of the propagator $P\left(\mathbf{q}, t \mid \mathbf{q}^{0}\right)$. The order up to which the short time approximation remains Gaussian depends on the particular form of the drift and diffusion coefficients. Moreover, the result does depend on the discretization scheme for the various stochastic integrals

$$
\begin{gathered}
\int_{0}^{\tau} d s f_{i}(s) W_{i j}^{k}(s), \\
W_{i j}(\tau)=\int_{0}^{\tau} d t_{j} \int_{0}^{t_{j}} d t_{j-1} \cdots \int_{0}^{t_{2}} d t_{1} \int_{0}^{t_{1}} d t_{0} f_{i}\left(t_{0}\right),
\end{gathered}
$$

involved in the expansion due to the dependence of $\mathbf{B}$ on Q. The calculation can be substantially simplified if one follows, without loss of generality, the Ito interpretation. In this case, just a few integrals do contribute to the averages. The propagator corresponding to the Stratonovich interpretation of Eq. (19) is then obtained from the ob- servation that this equation is the same as the Ito stochastic equation but with the drift vector $G_{i}$ replaced by $G_{i}+\frac{1}{2} B_{k j} \partial_{k} B_{i j}$.

We acknowledge the support of the Dirección General de Investigación Científica y Técnica of Spain for financial support (A. N. D.) and for Project No. PB95-0536 (M. M.).

*Permanent address: Institute for High Temperatures, 13/19 Izhorskaya Street, 127412, Moscow, Russia.

[1] R.P. Feynman and A. R. Hibbs, Quantum Mechanics and Path Integrals (McGraw-Hill, New York, 1965); R.P. Feynman, Statistical Mechanics (Benjamin, Reading, MA, 1972); L.S. Schulman, Techniques and Applications of Path Integration (Wiley, New York, 1981); F. Langouche, D. Roekaerts, and E. Tirapegui, Functional Integration and Semiclassical Expansions, Mathematics and Its Applications (Reidel, Dordrecht, 1982); H. Kleinert, Path Integrals in Quantum Mechanics, Statistics, and Polymer Physics (World Scientific, Singapore, 1995), 2nd ed.

[2] For a recent review, see the special issue on Functional Integration, J. Math. Phys. 36, No. 5 (1995).

[3] S. Chandrasekhar, Rev. Mod. Phys. 15, 1 (1943).

[4] H. Kramers, Physica (Utrecht) 7, 284 (1940).

[5] For recent reviews, see H. Risken, The Fokker-Planck Equation, Methods of Solution and Applications (Springer, New York, 1989); P. Hänggi, P. Talkner, and M. Borkovec, Rev. Mod. Phys. 62, 251 (1990); New Trends in Kramers' Reaction Rate Theory, edited by P. Talkner and P. Hänggi (Kluwer Academic Publishers, Dordrecht, 1995).

[6] A. N. Drozdov, Physica (Amsterdam) 196A, 258 (1993).

[7] R. M. Wilcox, J. Math. Phys. (N.Y.) 8, 962 (1967); H. De Raedt and B. De Raedt, Phys. Rev. A 28, 3575 (1983).

[8] M. Suzuki, J. Math. Phys. (N.Y.) 32, 400 (1991).

[9] A. N. Drozdov, Physica (Amsterdam) 196A, 283 (1993); Phys. Rev. Lett. 75, 4342 (1995); J. Chem. Phys. 105, 515 (1996).

[10] R. Phythian, J. Phys. A 10, 777 (1977); P. Hänggi, Z. Phys. B 75, 275 (1989); T. J. Newman, A. J. Bray, and A. J. McKane, J. Stat. Phys. 59, 357 (1990); A. J. Bray, A. J. McKane, and T.J. Newman, Phys. Rev. A 41, 657 (1990).

[11] See, e.g., R. L. Honeycutt, Phys. Rev. A 45, 600 (1992); 45, 604 (1992).

[12] K. Binder and D. W. Heermann, Monte Carlo Simulation in Statistical Physics (Springer, Berlin, 1988).

[13] For a review, see R. Kosloff, J. Phys. Chem. 92, 2087 (1988). 